\title{
Preface
}

\section{Recent Innovations on Biomedical Engineering}

This special supplement presents 50 papers selected from the proceedings of the International Conference on Biomedical Engineering Innovation 2015 (ICBEI2015) which was held from October 31 to November 03, 2015, at Eda World in southern Taiwan. ICBEI2015 provides relevant and practical information about this exciting and thriving new technology, and its various applications to Biomedical Engineering.

All of the papers submitted to the conference were first subject to examination by at least two independent reviewers prior to the conference. These selected papers subsequently underwent a rigorous peer-review process. Acceptance into this Special Issue was based on completion of the reviewers recommended and mandatory change and final examination by the Guest Editor. It is expected to be a key issue related to recent engineering innovations for Biomedical Engineers and academic researchers.

Lastly, I would like to sincerely thank all contributed authors and reviewers. I would also like to acknowledge the secretariats of the journal who helped with the process of publishing this issue. Special thanks must be given to the Editor-in-Chief for his kind support to the publication of this issue.

Guest Editor

Wen-Hsiang Hsieh

Professor

Department of Automation Engineering National Formosa University, Taiwan 